\title{
Detection of central circuits implicated in the formation of novel pain memories
}

\author{
Jaymin Upadhyay' \\ Julia Granitzka' \\ Thomas Bauermann² \\ Ulf Baumgärtner ${ }^{3}$ \\ Markus Breimhorst ${ }^{1}$ \\ Rolf-Detlef Treede ${ }^{3}$ \\ Frank Birklein' \\ 'Department of Neurology, \\ ${ }^{2}$ Department of Neuroradiology, \\ University Medical Centre, Johannes \\ Gutenberg University Mainz, Mainz, \\ ${ }^{3}$ Department of Neurophysiology, \\ Center for Biomedicine and Medical \\ Technology Mannheim (CBTM), \\ Heidelberg University, Mannheim, \\ Germany
}

This article was published in the following Dove Press journal: Journal of Pain Research

15 September 2016

Number of times this article has been viewed
Correspondence: Frank Birklein Universitätsmedizin, Klinik und Poliklinik für Neurologie, Johannes GutenbergUniversität Mainz, I Langenbeckstrasse, Mainz 55I3I, Germany

Tel $+49613 \mid 175486$

Fax +49 6131175625

Email Frank.Birklein@unimedizin-mainz.de

\begin{abstract}
Being able to remember physically and emotionally painful events in one's own past may shape behavior, and can create an aversion to a variety of situations. Pain imagination is a related process that may include recall of past experiences, in addition to production of sensory and emotional percepts without external stimuli. This study aimed to understand 1) the central nervous system processes that underlie pain imagination, 2) the retrieval of pain memories, and 3 ) to compare the latter with visual object memory. These goals were achieved by longitudinally investigating brain function with functional magnetic resonance imaging in a unique group of healthy volunteers who had never experienced tooth pain. In these subjects, we compared brain responses elicited during three experimental conditions in the following order: imagination of tooth pain (pain imagination), remembering one's own house (object memory), and remembrance of tooth pain following an episode of induced acute tooth pain (pain memory). Key observations stemming from group-level conjunction analyses revealed common activation in the posterior parietal cortex for both pain imagination and pain memory, while object and pain memory each had strong activation predominantly within the middle frontal gyrus. When contrasting pain imagination and memory, significant activation differences were observed in subcortical structures (ie, parahippocampus - pain imagination > pain memory; midbrain - pain memory > pain imagination). Importantly, these findings were observed in the presence of consistent and reproducible psychophysical and behavioral measures that informed on the subjects' ability to imagine novel and familiar thoughts, as well as the subjects' pain perception.
\end{abstract}

Keywords: fMRI, pain memory, pain imagination, object memory, tooth pain

\section{Introduction}

Memory is a key variable for pain management. ${ }^{1-3}$ On one hand, adequate memory and remembrance of painful events are of importance for individual history, classification, and proper treatment of pain. On the other hand, lively remembrance of previous painful events may influence future pain experiences by altering expectations, early emotional evaluation of pain stimuli, and cognitive processes. ${ }^{4-7}$

To address the issue of pain memory in more detail, subtypes have to be differentiated. Like other forms of memory, pain memory comprises two different principal types: implicit memory and explicit memory. ${ }^{8}$ Implicit memory, also referred to as nondeclarative memory, consists of a range of simple reflexes to complex behaviors, as well as of associative and nonassociative learning and memory (habituation and/ or sensitization to repetitive stimulation). ${ }^{9}$ Moreover, associative implicit memory is hypothesized to contribute to the development of chronic low-back pain. ${ }^{10}$ 
Explicit memory is declarative memory, and it includes semantic memory about facts and episodic memory about events. Some studies have investigated the remembrance and memory of ongoing clinical pain. In most studies, the reliability of pain remembrance is investigated in chronic patients suffering from arthritis ${ }^{11}$ or headache. ${ }^{12}$ Conversely, the impact of pain on memory in chronic pain patients has been examined, and there is general agreement that pain has detrimental effects on memory performance. ${ }^{13-16}$ Pain affects both the encoding and retrieval of common explicit memory, ${ }^{17}$ but whether it has an impact on the memory of pain itself is not known either. Scientific evaluation of the reliability and validity of pain memory in controlled experimental studies has just begun. ${ }^{18}$

This hypothesis-generating study was conducted to understand the brain responses that are involved in the generation of an explicit memory for pain. In order to address specificity for pain remembrance (explicit memory), we contrasted pain imagination and visual object remembrance in a within-subject design. We took the opportunity to investigate volunteers before (pain imagination) and after (pain memory) the application of experimental tooth pain. Given earlier observations in functional imaging studies probing interoception and affective components of pain, it was hypothesized that the insula cortex would be implicated during recall of pain, be it imagined or memory-based. ${ }^{19,20}$ Moreover, explicit memory requires the function of the parietal, temporal, and frontal cortices, as well as subcortical structures (eg, amygdala and hippocampus). ${ }^{21}$ As a result, these cortical regions were projected to drive the shaping of pain memory. The results presented herein contribute to the understanding of central correlates underlying pain-associated memory, as well as provide a comparison of brain function between pain memory and pain imagination.

\section{Materials and methods}

\section{Study participants}

A total of 12 healthy, right-handed male subjects (mean \pm standard deviation $23 \pm 2.56$ years of age) participated in this study. Study participants were recruited from the Johannes Gutenberg University Hospital medical student population. This study was approved by the ethics committee of the State Medical Association of Rhineland-Palatinate. Each subject gave informed written consent prior to participating in this study.

The inclusion criterion for the subjects was that each individual had no prior experience of tooth pain and no conscious memories of it. Given the uniqueness of this trait, subject recruitment was a limiting factor of this study, which should be considered when interpreting the results. Subjects were also required to have no history of migraine, increased incidence of headaches, depression, or existence of other neurological diseases. Furthermore, exclusion criteria included a presence of diabetes mellitus, disturbance of pain perception (eg, hypo- or hyperparesthesia or allodynia), disturbances of cognition, diseases consisting of pain symptoms (eg, inflammation or herniated disc), and contraindications for undergoing magnetic resonance imaging (MRI; eg, claustrophobia or metallic foreign body).

\section{Study design}

This investigation consisted of four study site visits by each subject. Between visits 1 and 2 were exactly 7 days; 5 months later, visit 3 and 7 days later visit 4 took place. Visit 1 consisted of an initial introduction to and training on a thought experiment, subsequently performed during functional MRI (fMRI; data collection). On visit 2, fMRI study session 1 was conducted, where the imagination of never-before-experienced tooth pain was investigated, in addition to remembrance of one's own house. Visit 3 comprised administration of experimental tooth pain (ice spray) to the middle incisors, and at visit 4 fMRI study session 2 was conducted, where the remembrance of the tooth pain experienced during visit 3 was investigated, in addition to remembrance of one's own house. The task "remember your own house" requests a remembrance rather than an imagination task, and it contrasts the abstract sensation "pain" from the visual object "house". The remembrance of the subjects' own house was further utilized as an internal control task because it induces a well-described pattern of brain activation in fMRI (ie, activation of the fusiform gyrus), which can be used as a validity criterion. ${ }^{22}$

\section{Visit I}

During visit 1 , subjects completed questionnaires assessing their mood (mood scale) $)^{23}$ and levels of anxiety and depression (Hospital Anxiety and Depression Scale [HADS]). ${ }^{24}$ Subsequently, procedures pertaining to the fMRI-scanning session were reviewed and training given regarding the tooth-pain (imagination and memory) and object-memory tasks. A color-coded scheme presented on a monitor was used to instruct subjects to imagine or remember pain (blue), remember an object (black), or rest (white). Following the thought experiment, the vividness of what subjects visually imagined or remembered was assessed using the Vividness of Visual Imagery Questionnaire (VVIQ) ${ }^{25}$ and an adapted version for pain remembrance (VVIQ-P). In addition, the pain experience scale (Schmerzempfindungsskala [SES]) ${ }^{26}$ was assessed. 


\section{HADS}

Before the first thought experiment during visit 1, the HADS was used to examine the extent to which subjects were classified as anxious or depressed. For this purpose, seven questions relating to mental states of anxiousness and depression were asked. The answers here are based on the subject's state of mind during the last 7 days. Using a points scheme of $0-3$, an individual anxiety value and a value to inform on presence of a depressed state was calculated. High values indicated an anxious or depressive personality. Individuals with a depression value of 8 or more were not included in the study, while high anxiety levels did not lead to exclusion.

\section{Mood scale}

Questions were asked to assess six mood states. Subjects were asked to rank on a simple visual analog scale states of depressed and euphoric, active and passive, tired and awake, dissolved and tense, anxious and safe, and lastly, satisfied and dissatisfied. The mood scale was used to assess the mental states of the subjects in respect of activity level, vigilance, internal stress state, self-confidence, and satisfaction. The questionnaire was used to exclude changes of mood at the different visits (ie, visits 1-4).

\section{VVIQ}

To quantify and control for the ability for visual imagination of study participants, the VVIQ questionnaire was used. Here, subjects were given a questionnaire consisting of 16 tasks, and asked to describe situations or things they imagined. This task was performed with open eyes, because of the comparability to the fMRI-scanning condition. To assess further the level of what was imagined and also described, a 5-point scale was used, where a maximum score of 5 represented "just as clear as normal vision" and a minimum score of 1 corresponded to an "inability to recall an image in mind". A high score indicates a strong visual sense, while a low score indicates a correspondingly weaker visual imagination of the subject. Data were analyzed as percentages of the maximum value of 80 .

\section{VVIQ-P}

To assess a subjects' ability to imagine pain, the VVIQ was amended with questions according to the topic of pain. Ten questions were answered by subjects based on situations that are associated with a painful experience. A 5-point rating scale was implemented, where 5 corresponded to a strong imagination of pain and 1 corresponded to an inability to imagine pain. A high score referred to a good imagination of pain, while a low score corresponded to a weaker ability to imagine pain. During visits $2-4$, the VVIQ and the VVIQ-P were completed in each case after the thought experiments or after the fMRI-scanning sessions. Data were analyzed as percentages of the maximum value of 80 .

\section{SES}

The SES was conducted in order to obtain more detail on the quality of pain imagined or remembered during visits 2 and 4. The SES questionnaire consists of 24 items, 14 of which pertain to the affective component of the pain experience and ten of which probe sensory properties of pain. Subjects were asked to rate how accurate each item or statement described their pain on a scale of 1 ("disagree") to 4 ("is exactly"). A calculation of a summed score of the affective and sensory items was then made.

\section{Visits 2 and 4}

Each fMRI scan consisted of 24 blocks consisting of three conditions: baseline (resting phase), pain (imagination [visit 2] or memory [visit 4] of a tooth pain), and control variable (object memory, ie, memory of subject's own house [visits 2 and 4]). The three conditions were presented in six 30 -second blocks, where a rest-period block bound each task block. The order of the task blocks was randomized between subjects, but held constant between fMRI-scan sessions 1 and 2 for each subject. Following the fMRI-scan sessions, subjects completed the mood scale (before and after scanning), VVIQ, VVIQ-P, and the SES. All subjects confirmed having performed the right task after the different cues (blue, black, white screen).

\section{Visit 3}

A week prior to visit 4, subjects experienced tooth pain for the first time. Tooth pain was accomplished with ice spray (polar ice spray at $-45^{\circ} \mathrm{C}$; Unigloves). The ice spray was applied for 1 second to the centerline on one of the front upper incisors. Cold stimulation of a tooth provokes an intense $(>8 / 10$ in any case) tearing pain, which lasts for a few seconds. The stimulus was not repeated.

\section{MRI-data acquisition}

All MRI data were collected on a 1.5 T Siemens Magnetom Sonata scanner with an eight-channel head coil. fMRI data were collected using an echo-planar imaging pulse sequence consisting of the following parameters: repetition time 3,050 milliseconds; echo time (TE) 50 milliseconds; scan duration 12 minutes, 12 seconds; number of slices 
36; slice thickness $3 \mathrm{~mm}$; and field of view $192 \times 192$ $\mathrm{mm}^{2}$. High-resolution anatomical MRI was also collected (magnetization-prepared rapid gradient-echo imaging) with the following parameters: repetition time 2,160 milliseconds; echo time 3.39 milliseconds; inversion time 1,100 milliseconds; scan duration 10 minutes, 6 seconds; number of averages 2; 3-D block thickness $176 \mathrm{~mm}$; and field of view $256 \times 256 \mathrm{~mm}^{2}$.

\section{Data analysis}

\section{Psychometric data analysis}

Analyses of psychometric data were performed with SPSS 15.0 for Windows (SPSS Inc., Chicago, IL, USA). HADS, mood questionnaire, VVIQ, VVIQ-P, and SES data were analyzed descriptively, and are presented herein with mean and standard deviation. Determination of statistically significant differences was carried out using analysis of variance for the HADS and mood questionnaire, while two-tailed paired $t$-tests were used for all other psychometric data. Significant results were defined as $P<0.05$.

\section{MRI-data analysis}

MRI-data analysis was performed using a combination of in-house MatLab scripts, SPM5 and MRIcro. Singlesubject preprocessing steps consisted of motion correction, creation of the mean functional image for registration to the Montreal Neurological Institute standard brain template for group-level analyses, spatial smoothing $\left(8 \times 8 \times 10 \mathrm{~mm}^{3}\right.$ smoothing kernel), and intensity normalization using a Gaussian distribution model. To determine task-dependent brain activation within each fMRI data set, blocks of pain imagination, pain memory, and object memory were each contrasted against the rest period. Here, a general linear model approach was used.

At the group level, statistical maps for each scan session were calculated and statistically compared using two-tailed paired $t$-tests to identify brain activity corresponding to pain imagination, pain memory, and object memory. Due to the limited availability of participants fulfilling our requirements, the threshold value for group-level statistical maps was set to $P<0.001$ (uncorrected); however, a cluster-size threshold of five voxels was utilized throughout. Conjunction analyses were performed to determine common activity between pain imagination and pain memory, as well as pain memory and object memory. The conjunction maps were tested against the global null hypothesis $(P<0.001$, uncorrected) and also with a cluster-size threshold of five voxels.

\section{Results \\ Psychometric results}

The HADS questionnaire was used as selection criteria for this study. Participants of this study had a mean \pm standard deviation HADS anxiety value of $4.3 \pm 2.95$ and a HADS depression mean \pm standard deviation value of $1.7 \pm 1.95$, which were all in the nonpathological range. With respect to the mood questionnaire, significant differences were not detected between individual scanning sessions $\left(F_{1,9}=0.18, P=0.68\right)$, pre- and postmeasurement during each scan session, $\left(F_{1,9}=2.948\right.$, $P=0.12)$, or an interaction of factors $\left(F_{1,9}=0.11, P=0.75\right)$, all indicating stable mood throughout the duration of the study. Psychometric data were not available for one subject.

Vividness ratings of visual object imagination (wellknown object) were high and equal in both fMRI sessions (visit $1,78.1 \% \pm 12.1 \%$; visit $2,78.2 \% \pm 12.6 \%$; visit 3 , $76.2 \% \pm 13.1 \%$; Figure S1). Vividness ratings of pain imagination and remembrance (tooth pain, unknown to the subjects) were also high (visit $1,64.5 \% \pm 15.9 \%$; visit $2,65.7 \% \pm 14.4 \%$; visit $3,63.9 \% \pm 14.1 \%$; Figure S1), indicating that medical students had acquired enough cognitive knowledge about this pain state without having experienced it themselves. At any time point, the vividness rating for visual objects was higher than for pain (see Figure S1 for details). During the second MRI-scanning session, when subjects remembered an actual experience of tooth pain, vividness of remembered pain did not differ from that of the previously imagined pain $(t=0.689, d f=9 ; P=0.508)$. Also, the sensory $(t=-0.05, d f=9$; $P=0.97)$ and the affective $(t=0.802, d f=9 ; P=0.443)$ paindescriptor ratings of the SES were constant across sessions (Figure S2), with hammering, throbbing, stinging, and cutting ratings having the biggest numerical differences. At the single-descriptor level, the value for "cutting" increased from visits 2 to 4 ( $P<0.05$, Wilcoxon test).

\section{Pain imagination (visit 2) and memory (visit 4)}

The imagination of tooth pain (midline at the incisors) by healthy subjects that had never experienced this type of pain elicited significant activation in a network of cortical structures, including the anterior insula, inferior parietal lobe (posterior parietal cortex), and inferior frontal gyrus (Figure 1A, Table S1). This activation during the pain-imagination task was primarily unilateral (left side). Following the experience of tooth pain, which occurred during visit 3 , subjects were able to perform a pain-memory task (remembrance). Common brain activation was observed between pain imagination 

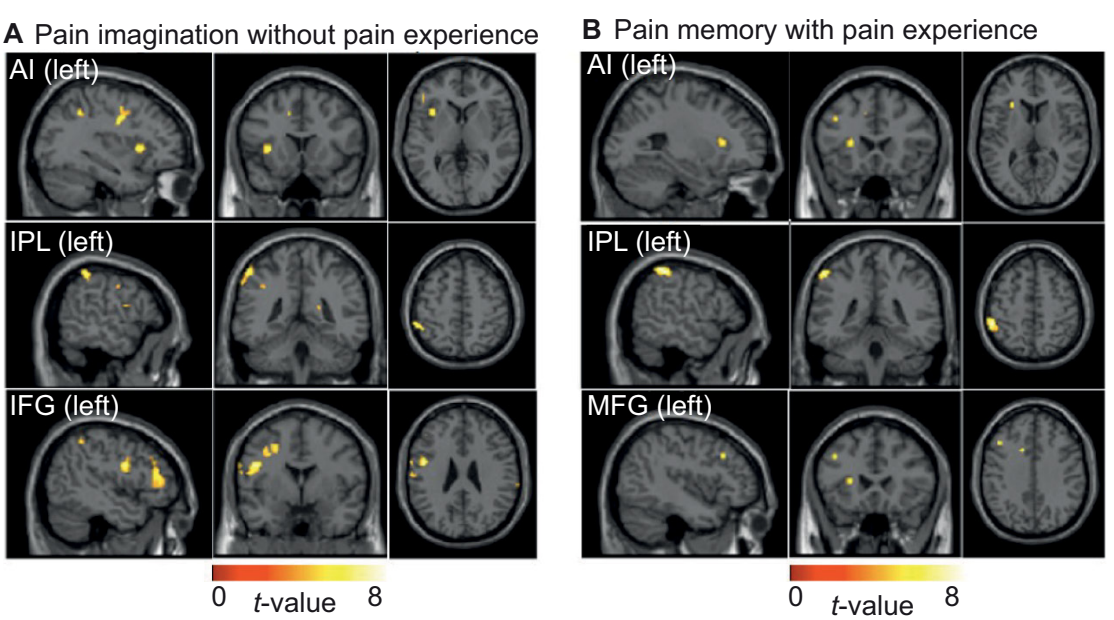

C Pain imagination vs pain memory

Pain imagination > pain memory

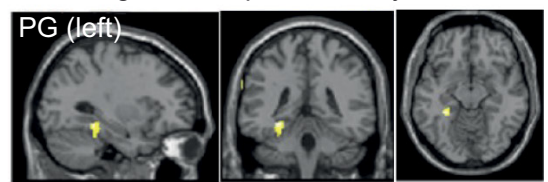

Pain imagination < pain memory

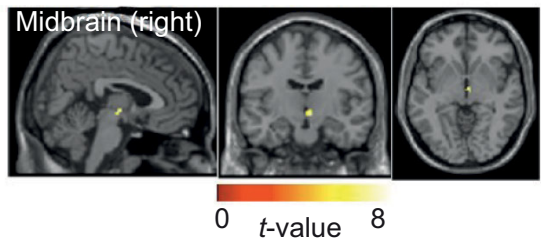

Figure I Brain activation during pain imagination (visit 2) and pain memory (visit 4).

Notes: (A) Prior to administration of tooth pain, the imagination of tooth pain induced activation in network of cortical structures. (B) The remembrance of tooth pain elicited a very similar cortical activation pattern in comparison to the pain imagination task. $(\mathbf{C})$ BOLD responses of greater magnitude were detected during tooth pain imagination in deeper brain regions such as the PG, while a greater midbrain response was measured during pain memory. See Table SI for summary statistics corresponding to BOLD activation maps. Abbreviations: BOLD, blood oxygen level-dependent; Al, anterior insula; IFG, inferior frontal gyrus; IPL, inferior parietal lobe; MFG, middle frontal gyrus; PG, parahippocampal gyrus.

and memory (Figure 1B, Table S1) in such regions as the anterior insula. However, a statistical comparison between the two conditions demonstrated a higher level of activity in the parahippocampal gyrus during the pain-imagination condition (Figure 1C upper panel, Table S1), while increased activation in the midbrain was observed during pain memory (Figure 1C lower panel, Table S1).

\section{Object memory (visits 2 and 4)}

There was no difference in activation of any brain region between the results of object memory during visits 2 and 4. The results are thus reported together. During the object (one's own house)-memory task, robust brain activity was observed in cortical and subcortical brain structures (Figure 2, Table S2). The activation pattern observed during this control task was in good accord with previous work probing the figural memory system. ${ }^{22} \mathrm{~A}$ comparison between pain imagination and object memory (Table 1), as well as pain memory and object memory, showed that remembrance of one's own house elicited much greater activation in most brain regions. One notable exception to this trend was observed within the inferior parietal lobe, where greater activation was observed during pain memory than object memory (Table 1).

\section{Conjunction analyses}

Conjunction analysis between pain imagination and pain memory yielded common activation to be statistically observed solely in the left inferior parietal lobe (Figure 3A, Table S3). Similar procedures performed between the pain-memory and house-memory conditions yielded common brain activity, primarily within the middle frontal

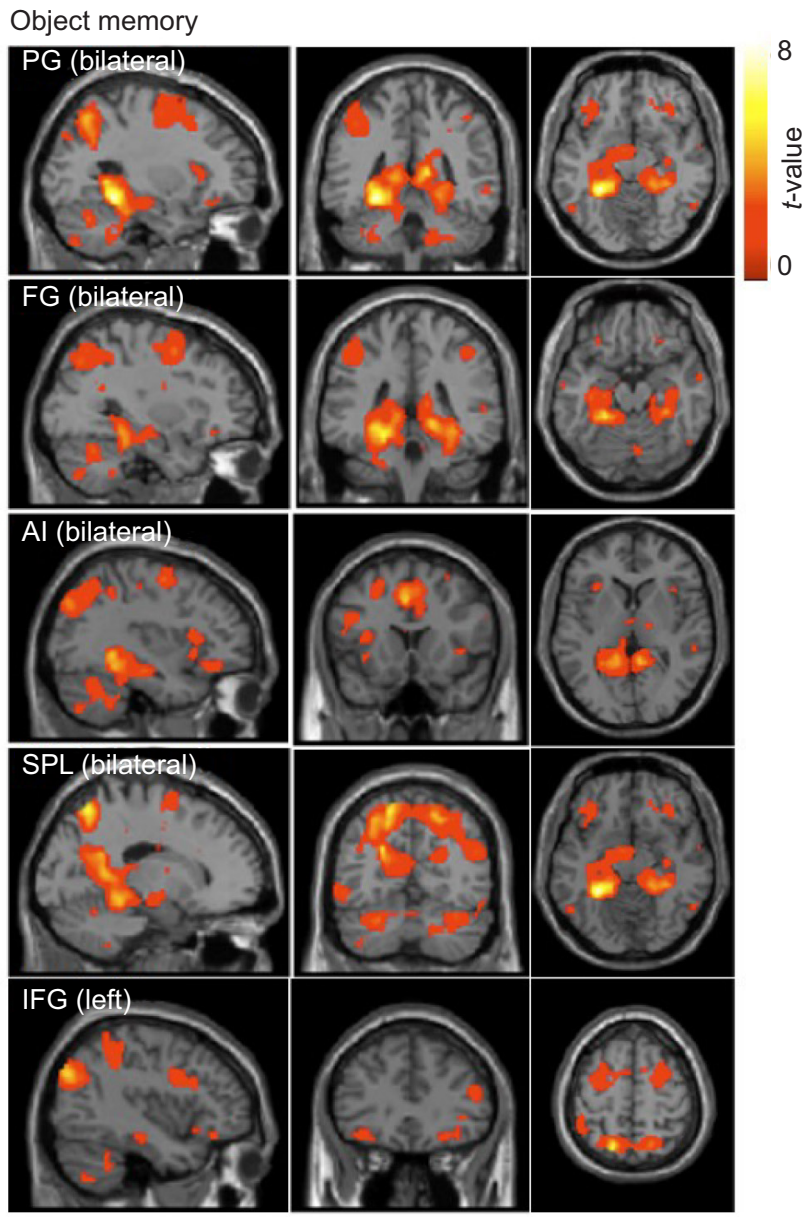

Figure 2 Brain activation during object memory (visits 2 and 4).

Notes: fMRI data stemming from the object (ie, house) memory task performed during visits 2 and 4 were combined for group analyses. Memory of one's own house induced robust brain activation in a number of cortical and subcortical brain regions. See Table S2 for summary statistics corresponding to BOLD activation maps. Abbreviations: $\mathrm{fMRI}$, functional magnetic resonance imaging; BOLD, blood oxygen level-dependent; PG, parahippocampal gyrus; FG, fusiform gyrus; Al, anterior insula; SPL, superior parietal lobule; IFG, inferior frontal gyrus. 
Table I Group level $(n=10)$ brain activation comparing pain imagination vs house memory and pain memory vs object memory

\begin{tabular}{|c|c|c|c|c|c|}
\hline \multicolumn{6}{|c|}{ Pain imagination vs house memory } \\
\hline t-Contrast & Brain area & BA & $x, y, z$ & $\begin{array}{l}\text { Cluster size, } \\
\text { voxel }\end{array}$ & t-Value \\
\hline \multicolumn{6}{|l|}{ Pain imagination < object memory } \\
\hline \multirow[t]{10}{*}{ Activations - right hemisphere } & Parahippocampal gyrus & 36 & $24,-34,-16$ & 90 & 6.21 \\
\hline & Precuneus & 7 & $4,-68,56$ & 121 & 6.15 \\
\hline & Posterior cingulate & 23,29 & $6,-42,8$ & 145 & 6.04 \\
\hline & Corpus callosum & & & 60 & \\
\hline & Cerebellar tonsil & & $14,-52,-48$ & 51 & 5.72 \\
\hline & Superior parietal lobule & 7 & $38,-64,52$ & 39 & 5.34 \\
\hline & Precuneus and cingulate gyrus & 7 & $4,-40,46$ & 35 & 4.95 \\
\hline & Precuneus and angular gyrus & 19,39 & $42,-76,44$ & 17 & 4.92 \\
\hline & Cerebellum anterior lobe, culmen & & $10,-62,-12$ & 9 & 4.87 \\
\hline & Middle frontal gyrus & 8 & $30,36,50$ & 9 & 4.71 \\
\hline \multirow[t]{14}{*}{ Activations - left hemisphere } & Precuneus & 19 & $-38,-78,42$ & 86 & 10.56 \\
\hline & Middle temporal gyrus & 39 & & 161 & 6.79 \\
\hline & Angular gyrus & & & 67 & \\
\hline & Thalamus & & $-22,-30,0$ & 33 & 10.12 \\
\hline & Pulvinar & & & 12 & \\
\hline & Limbic and temporal lobe & 37 & $-28,-42,-16$ & 190 & 8.14 \\
\hline & Fusiform gyrus & 37 & & 26 & 8.14 \\
\hline & Parahippocampal gyrus & 19 & & 164 & 6.73 \\
\hline & Posterior cingulate & 30 & $-10,-50,14$ & 241 & 7.39 \\
\hline & Cerebellar tonsil & & $-26,-46,-46$ & 47 & 7.09 \\
\hline & Pyramis and inferior semilunar lobule & & $-34,-74,-44$ & 24 & 6.36 \\
\hline & Cerebellum posterior lobe, pyramis & & $-18,-82,-40$ & 8 & 5.44 \\
\hline & Cerebellar tonsil & & $-38,-60,-42$ & 20 & 5.43 \\
\hline & Cerebellum posterior lobe, declive & & $-30,-64,-26$ & 6 & 4.89 \\
\hline \multicolumn{6}{|c|}{ Pain memory vs object memory } \\
\hline t-Contrast & Brain area & BA & $x, y, z$ & Cluster size & $t$-Value \\
\hline \multicolumn{6}{|l|}{ Pain memory > object memory } \\
\hline Activations - left hemisphere & Inferior parietal lobule & & $-54,-50,54$ & 5 & 5.69 \\
\hline \multirow[t]{19}{*}{ Activations - right hemisphere } & Temporal, parietal, and limbic lobes & & $22,-52,28$ & 233 & 7.72 \\
\hline & Posterior cingulate & & & 92 & 5.5 \\
\hline & Corpus callosum & & & 42 & \\
\hline & Precuneus & 7 & $18,-62,52$ & 136 & 7.19 \\
\hline & Superior parietal lobule & 7 & & 51 & \\
\hline & Middle temporal gyrus & 37 & $64,-58,-6$ & 8 & 6.72 \\
\hline & Parahippocampal gyrus & 36,37 & $34,-36,-12$ & 36 & 6.62 \\
\hline & Fusiform gyrus & 37 & & 8 & \\
\hline & Middle temporal gyrus & 39 & $52,-72,30$ & 27 & 6.42 \\
\hline & Pons & & $12,-36,-34$ & 17 & 6.1 \\
\hline & Middle temporal gyrus (temporal pole) & 21 & $56,10,-18$ & 14 & 5.82 \\
\hline & Parahippocampal gyrus & 30 & $22,-46,-2$ & 45 & 5.82 \\
\hline & Frontal lobe & 6 & $26,-10,56$ & 22 & 5.75 \\
\hline & Middle frontal gyrus & 6 & & 8 & 5.75 \\
\hline & Precentral gyrus (arm/hand) & & & 5 & \\
\hline & Precuneus & 7 & $6,-68,60$ & 10 & 5.02 \\
\hline & Precuneus and paracentral lobule & 5,7 & $4,-46,54$ & 12 & 4.89 \\
\hline & Paracentral lobule & 5 & $4,-38,50$ & 8 & 4.76 \\
\hline & Middle frontal gyrus & 6 & $28,0,60$ & 13 & 4.72 \\
\hline \multirow[t]{5}{*}{ Activations - left hemisphere } & Limbic and temporal lobes & $19,20,36,37$ & $-30,-44,-12$ & 398 & 12.1 \\
\hline & Parahippocampal gyrus & $19,36,37$ & & 278 & 12.1 \\
\hline & Fusiform gyrus & 20 & & 120 & \\
\hline & Frontal lobe & 6 & $-20,-8,66$ & 40 & 8.32 \\
\hline & Middle frontal gyrus & 6 & & 17 & 8.32 \\
\hline
\end{tabular}




\begin{tabular}{|c|c|c|c|c|c|}
\hline \multicolumn{6}{|c|}{ Pain memory vs object memory } \\
\hline$t$-Contrast & Brain area & BA & $x, y, z$ & Cluster size & t-Value \\
\hline & Temporal, parietal, and limbic lobes & & $-24,-58,22$ & 158 & 7.72 \\
\hline & Posterior cingulate & & & 40 & 5.45 \\
\hline & Corpus callosum & & & 27 & \\
\hline & Precuneus & 19,39 & $-38,-80,34$ & 112 & 6.7 \\
\hline & Angular gyrus & 39 & & 91 & \\
\hline & Middle temporal gyrus & & & 47 & \\
\hline & Postcentral gyrus (hand/eye) & 2 & $-46,-34,60$ & 17 & 5.97 \\
\hline & Cerebellar tonsil & & $-12,-56,-50$ & 12 & 4.48 \\
\hline
\end{tabular}

Abbreviation: BA, Brodman area.

A Conjuction analysis: Pain imagination + pain memory

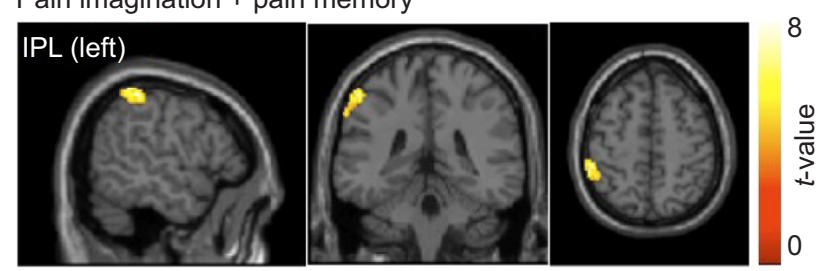

B Conjuction analysis:

Pain memory + object memory

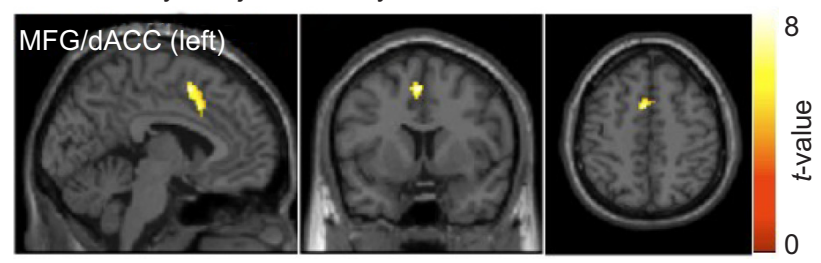

Figure 3 Conjunction analyses of $\mathrm{PMRI}$ data.

Notes: Conjunction analyses were performed in order to determine common activation between pain imagination and pain memory $(\mathbf{A})$ and between pain memory and object memory (B) While the imagination and remembrance of pain each activated the left IPL, remembering tooth pain and one's own house activated a cluster with the MFG and dACC. See Table S3 for summary statistics corresponding to BOLD activation maps. Abbreviations: $\mathrm{FMRI}$, functional magnetic resonance imaging; IPL, inferior parietal lobe; MFG, middle frontal gyrus; $\mathrm{AACC}$, dorsal anterior cingulate cortex; BOLD, blood oxygen level-dependent.

gyrus (Figure 3B, Table S3). No other common statistically significant clusters of activation or deactivation for the latter conjunction analysis were observed.

\section{Discussion}

In the current fMRI study, the central responses associated with forming an explicit memory of pain were characterized in a healthy subject population possessing no prior experience of tooth pain. Given the activation observed within the anterior insula, inferior parietal lobule, and middle frontal gyrus during remembrance of a prior painful experience (ie, cold-induced tooth pain) in conjunction with the differential parahippocampus activation observed during pain imagination versus pain memory, a network of structures implicated in pain-associated memory formation were elucidated. Imagination of motor action is much better investigated than investigation of sensory phenomena like pain. Since imagination of movement alone is similarly effective to motor exercise to influence motor networks after stroke, ${ }^{27}$ we are confident that pain-associated memory-formation activation patterns correspond to function within a specific central nervous system network, although this cannot be concluded solely from the current data set. However, earlier resting-state fMRI findings have reported functional connectivity among the inferior parietal lobule with the middle frontal gyrus and greater default-mode network, ${ }^{28}$ while the anterior insula connects more to other limbic system structures, such as the cingulate and frontal cortex. ${ }^{29}$

Interestingly, our investigation did not yield activation or deactivation patterns within the somatosensory system, suggesting more limbic or affective pain centers being implicated in pain memory. This latter observation is somewhat in contrast to earlier work. However, while it was long hypothesized that action execution, imagery, and observation were functionally equivalent, a very recent study investigating visual and kinesthetic motor imagination failed to demonstrate activation of the primary motor cortex,${ }^{30}$ similar to pain imagination and pain memory failing to activate the primary sensory cortices in our study. What remains to be determined in subsequent work is whether previous and current observations in healthy volunteers translate to a chronic pain setting where specific pain memories likely alter patients' behavior(s). Whether pain is imagined or recollected, negative affect can be a relevant feature of either process, ${ }^{31}$ necessitating the involvement of limbic system structures (ie, insular, frontal, and cingulate cortices), of which there is substantial overlap with circuitry enabling attention and memory. ${ }^{32-34}$ Moreover, differences in activation observed within the parahippocampus when contrasting pain memory from pain imagination may have been derived from internally verbalizing the earlier induction of pain. ${ }^{35}$ In this specific study population, as salience of a painful event is gained, the change in functionality of cortical and subcortical regions may be indicative of not only memory formation but also the semantics an individual utilizes to recall and describe a past painful experience. 
It has been noted that the activation patterns during pain imagination and memory overlap with some (ie, anterior insular and mid-cingulate) but not all (eg, thalamus and primary somatosensory cortex) brain regions activated, especially during bottom-up processing of nociceptive stimuli. ${ }^{36}$ Work performed by Fairhurst et al similarly probed brain correlates of recall of noxious heat using fMRI. ${ }^{37}$ Here, common activation during the actual pain stimulation and pain recall was indeed observed in such structures as the cerebellum, thalamus, and somatosensory cortex, a result that may stem from the stimuli themselves or the short duration of time between stimulus delivery and recall (11 seconds).

\section{Behavioral validation procedures and outcomes}

In addition to commonly utilized assessments of mental health (ie, HADS), metrics informing on the subjects' mood, imaginative ability (VVIQ and VVIQ-P), and pain perception (SES) were measured throughout the study. First, all study volunteers possessed and maintained good mental health during visits $1-4$. Based on the VVIQ and VVIQ-P questionnaires, consistent levels of imaginative ability, particularly during visits 2 and 4, were importantly observed. It may be expected that the VVIQ-P would be altered at visit 4 compared to visit 2 , given the introduction of tooth pain at visit 3 . However, it must be noted that the VVIQ-P was in the context of perception of pain in general, rather than of the particular tooth pain. Moreover, the design of our study must have had order (carryover) effects. It is likely that pain ratings of pain memory and recall may have differed if the order of the sessions could have been changed. However, the fact that there was no difference in the imaging results of object memory between sessions 2 and 4 suggests that these order effects might be of minor importance. Vividness memories were generally higher for visually based memories than those related to pain. This finding in healthy subjects was believed to stem from more familiarity of one's own home, a common and frequently encountered object, compared to remembrance of pain (at best, of tooth pain), an experience encountered sporadically in healthy volunteers. This result could differ in chronic pain patients, where the pain experience is a frequent and robust occurrence.

Similarly, SES global values for both sensory and affective components remained in large part unchanged between visits 2 and 4 . This was surprising, because one would expect at least changes in the sensory component between tooth pain imagination and remembrance. However, this result might be technical, given that pain-component scores were sum scores. Some values went up between the sessions, and some went down, resulting in no change. Supporting this interpretation, the sensitivity analysis of single items of the sensory component revealed that the description "cutting" was rated more highly after tooth-pain experiences. Alternatively, it has been repetitively shown that nonfigural remembering, such as voices, is much more blurred than figural remembering, such as faces. ${ }^{38}$ One could speculate that pain might be too abstract to recall reliably the different components of a particular pain some days ago. The subjects might have reported a general summary of their previous pain experiences, instead of the specific facets of tooth pain. This is different if the magnitude of pain is recalled, ${ }^{39}$ which was however not in the scope of our study.

By incorporating the object-memory task within the experimental paradigm, two key and related objectives were met: one, a known template of memory circuitry was obtained for comparative purposes (ie, pain memory vs object memory); and two, given the agreement between central structures activated in the current study with earlier work, ${ }^{22}$ the objectmemory and imagination capabilities of the current study population were deemed intact from an fMRI perspective.

\section{Cortical representation of pain imagination and memory}

In light of the emotional and interoceptive components of imagining or recollecting a painful experience, it is not surprising that such structures as the anterior insula and frontal cortices were activated during pain imagination and memory. ${ }^{40-44}$ Interestingly, imagery of pain and itch by healthy subjects elicited blood oxygen level-dependent responses in the anterior insula. ${ }^{45}$ Within the middle frontal gyrus specifically, a cluster of activation was commonly activated by the pain-memory and object-memory tasks. Activity in this structure was likely driven by episodic retrieval and may have involved context-dependent memory, given retrieval of the circumstances under which tooth pain was administered for pain memory and the setting or environment of one's own house.

A slightly less expected result was the activation of the inferior parietal lobule (posterior parietal cortex) during pain imagination and memory, yet greater activation in this structure during pain compared to object memory. This result may relate to the fact that the parietal cortex in general is integral for localizing one's own body, a mental process needed for localizing pain within the body (intrapersonal imagination). ${ }^{44}$ The potential role of the inferior parietal lobule for pain memory was further suggested by the lack of common activation in this structure following conjunction analysis between pain and object memory. In a previous study, it was shown that the inferior parietal lobule is activated by touching one hand, ${ }^{46}$ while the left inferior parietal lobule 
specifically is activated if subjects watch the expression of pain in other faces. ${ }^{47}$ Therefore, it is speculated that the inferior parietal lobule-specific observation may reflect a similar central process to that present and observed during pain empathy ${ }^{48,49}$ or during processes engaging the mirrorneuron system. However, subjective measures supporting the presence of "self-empathy" were not obtained in this study.

\section{Subcortical representation of pain imagination and memory}

When brain-activation maps were contrasted between the pain-imagination and pain-memory conditions, the parahippocampus was observed to have greater activation during pain imagination. Prior fMRI work in healthy human subjects has suggested the role of the parahippocampus during imagined locomotor tasks, ${ }^{50}$ as well as representation of objects in 3-D spaces. ${ }^{51,52}$ The contrast analysis also revealed a significantly greater degree of midbrain (left hemisphere) activity during the pain-memory task. Previous results have not implicated such deep subcortical structures in imagination or memory, yet enhanced midbrain responses during nociception (eg, for pain control) are well established. ${ }^{53}$ If the midbrain activation during a pain-memory task is indeed derived from supraspinal nociceptive processes, it may be expected that any response detected at the midbrain level should remain ipsilateral to the site of evoked pain stimulation. Therefore, in future work, more lateralized pain stimulation (midline oral pain was induced in the current study) and subsequent potential detection of midbrain activity may help decipher the role of supraspinal nociceptive process in general during the formation of pain memories.

\section{Study limitations}

\section{Sample size}

Given the prevalence of tooth pain in the general population, the identification and recruitment of healthy subjects without a self-reported history of tooth pain proved to be very difficult. As a result, this study consisted of a small $(n=12)$ sample, with all the well-known shortcomings. In future work and in light of the ubiquity of tooth pain, an experimental condition involving pain induced by cuff algometry and reflecting pain perceived during ischemic-like claudication in peripheral arterial disease may be an experimental condition to consider.

\section{fMRI-data acquisition and analysis}

The current study was performed at $1.5 \mathrm{~T}$ and with an eightchannel receiver coil. This equipment-related limitation in conjunction with the same study population likely contributed to less robust fMRI results compared to what may be observed in a similar study performed at higher field strength, better head-coil sensitivity, and an n=15-20 cohort. Moreover, in order initially to determine brain responses associated with pain memory and imagination, this study only reports uncorrected, group-level fMRI results. In future work, particularly where a more appropriate sample size is achieved, a multiple-comparison procedure, such as cluster-size or false-discovery-rate correction, should be incorporated.

\section{Control condition}

The recollection of one's own house (ie, object-memory condition) was performed by participants in order to elucidate associative memory, and to control for appropriate participation in the mental tasks. Such a control condition can be considered a complex memory task that incorporates visuospatial, autobiographical, and spatial memories, which are not necessarily components of pain memory. In future fMRI work, experimental control conditions that may be beneficial to implement could include noxious sensory stimuli and comparison of imagining an unknown object presented at visit 2 and remembered at visit 4 .

\section{Conclusion}

By using a longitudinal design, this study characterized the central structure and networks underlying the formation of pain memories within a small, yet specialized healthy subject population. The "remembrance" of pain, be it based on a solely imaginative process or based on a true memory of a painful experience, appears to involve the inferior parietal lobule, yet the mechanism of forming a pain memory shares aspects of memory formation and retrieval of general episodic memories.

\section{Acknowledgments}

This study was supported by DFG Bi 579/8-1 and Tr 236/134, Foundation Rhineland-Palatinate (936). We gratefully thank Dr Hannele H Jantsch for her work on this study.

\section{Disclosure}

The authors report no conflicts of interest in this work.

\section{References}

1. Noel M, Chambers CT, Petter M, McGrath PJ, Klein RM, Stewart SH Pain is not over when the needle ends: a review and preliminary model of acute pain memory development in childhood. Pain Manag. 2012; 2(5):487-497.

2. Lithwick A, Lev S, Binshtok AM. Chronic pain-related remodeling of cerebral cortex - 'pain memory': a possible target for treatment of chronic pain. Pain Manag. 2013;3(1):35-45.

3. Vadivelu N, Mitra S, Narayan D. Recent advances in postoperative pain management. Yale J Biol Med. 2010;83(1):11-25. 
4. Sawamoto N, Honda M, Okada T, et al. Expectation of pain enhances responses to nonpainful somatosensory stimulation in the anterior cingulate cortex and parietal operculum/posterior insula: an event-related functional magnetic resonance imaging study. J Neurosci. 2000;20(19):7438-7445.

5. Keefe FJ, Dunsmore J, Burnett R. Behavioral and cognitive-behavioral approaches to chronic pain: recent advances and future directions. J Consult Clin Psychol. 1992;60(4):528-536.

6. Benedetti F, Pollo A, Lopiano L, Lanotte M, Vighetti S, Rainero I. Conscious expectation and unconscious conditioning in analgesic, motor, and hormonal placebo/nocebo responses. JNeurosci. 2003;23(10):4315-4323.

7. Lobanov OV, Zeidan F, McHaffie JG, Kraft RA, Coghill RC. From cue to meaning: brain mechanisms supporting the construction of expectations of pain. Pain. 2014;155(1):129-136

8. Klein T, Magerl W, Treede RD. Forget about your chronic pain. Pain. 2007;132(1-2):16-17.

9. Prescott SA. Interactions between depression and facilitation within neural networks: updating the dual-process theory of plasticity. Learn Mem. 1998;5(6):446-466.

10. Flor $\mathrm{H}$. The functional organization of the brain in chronic pain. Prog Brain Res. 2000;129:313-322.

11. Lefebvre JC, Keefe FJ. Memory for pain: the relationship of pain catastrophizing to the recall of daily rheumatoid arthritis pain. Clin $J$ Pain. 2002;18(1):56-63.

12. Kikuchi H, Yoshiuchi K, Miyasaka N, et al. Reliability of recalled selfreport on headache intensity: investigation using ecological momentary assessment technique. Cephalalgia. 2006;26(11):1335-1343.

13. Kuhajda MC, Thorn BE, Klinger MR. The effect of pain on memory for affective words. Ann Behav Med. 1998;20(1):31-35.

14. Grisart JM, Plaghki LH. Impaired selective attention in chronic pain patients. Eur J Pain. 1999;3(4):325-333.

15. Dick BD, Rashiq S. Disruption of attention and working memory traces in individuals with chronic pain. Anesth Analg. 2007;104(5):1223-1229.

16. Terry RH, Niven CA, Brodie EE, Jones RB, Prowse MA. Memory for pain? A comparison of nonexperiential estimates and patients' reports of the quality and intensity of postoperative pain. J Pain. 2008;9(4):342-349.

17. Kuhajda MC, Thorn BE, Klinger MR, Rubin NJ. The effect of headache pain on attention (encoding) and memory (recognition). Pain. 2002;97(3):213-221.

18. Gedney JJ, Logan H. Pain related recall predicts future pain report. Pain. 2006;121(1-2):69-76.

19. Craig AD. Interoception: the sense of the physiological condition of the body. Curr Opin Neurobiol. 2003;13(4):500-505.

20. Wiech K, Lin CS, Brodersen KH, Bingel U, Ploner M, Tracey I. Anterior insula integrates information about salience into perceptual decisions about pain. J Neurosci. 2010;30(48):16324-16331.

21. Schott BH, Henson RN, Richardson-Klavehn A, et al. Redefining implicit and explicit memory: the functional neuroanatomy of priming, remembering, and control of retrieval. Proc Natl Acad Sci USA. 2005;102(4):1257-1262.

22. Ishai A, Ungerleider LG, Haxby JV. Distributed neural systems for the generation of visual images. Neuron. 2000;28(3):979-990.

23. Stern RA. Assessment of mood states in neurodegenerative disease: methodological issues and diagnostic recommendations. Semin Clin Neuropsychiatry. 1996;1(4):315-324.

24. Zigmond AS, Snaith RP. The hospital anxiety and depression scale. Acta Psychiatr Scand. 1983;67(6):361-370.

25. Marks DF. Visual imagery differences in the recall of pictures. $B r J$ Psychol. 1973;64(1):17-24.

26. Geissner E. Dimensionen der Verarbeitung chronischer SchmerzenEine Replikationsstudie [Dimensions of coping with chronic pain: a replication study]. Z Klin Psychol Psychopathol Psychother. 1992;40(1):20-33.

27. Bajaj S, Butler AJ, Drake D, Dhamala M. Brain effective connectivity during motor-imagery and execution following stroke and rehabilitation. Neuroimage Clin. 2015;8:572-582.

28. Zhang S, Li CS. Functional clustering of the human inferior parietal lobule by whole-brain connectivity mapping of resting-state functional magnetic resonance imaging signals. Brain Connect. 2014;4(1):53-69.
29. Cauda F, D’Agata F, Sacco K, Duca S, Geminiani G, Vercelli A. Functional connectivity of the insula in the resting brain. Neuroimage. 2011;55(1):8-23.

30. Kilintari M, Narayana S, Babajani-Feremi A, Rezaie R, Papanicolaou A. Brain activation profiles during kinesthetic and visual imagery: an fMRI study. Brain Res. 2016;1646:249-261.

31. Rainville P. Brain mechanisms of pain affect and pain modulation. Curr Opin Neurobiol. 2002;12(2):195-204.

32. Uncapher MR, Rugg MD. Fractionation of the component processes underlying successful episodic encoding: a combined $\mathrm{fMRI}$ and dividedattention study. J Cogn Neurosci. 2008;20(2):240-254.

33. Uncapher MR, Rugg MD. Effects of divided attention on fMRI correlates of memory encoding. J Cogn Neurosci. 2005;17(12):1923-1935.

34. Reber PJ, Gitelman DR, Parrish TB, Mesulam MM. Dissociating explicit and implicit category knowledge with fMRI. J Cogn Neurosci. 2003;15(4):574-583.

35. Smith CN, Squire LR. Medial temporal lobe activity during retrieval of semantic memory is related to the age of the memory. $J$ Neurosci. 2009;29(4):930-938.

36. Apkarian AV, Bushnell MC, Treede RD, Zubieta JK. Human brain mechanisms of pain perception and regulation in health and disease. Eur J Pain. 2005;9(4):463-484.

37. Fairhurst M, Fairhurst K, Berna C, Tracey I. An fMRI study exploring the overlap and differences between neural representations of physical and recalled pain. PLoS One. 2012;7(10):e48711.

38. Damjanovic L, Hanley JR. Recalling episodic and semantic information about famous faces and voices. Mem Cognit. 2007;35(6):1205-1210.

39. Jantsch HH, Gawlitza M, Geber C, et al. Explicit episodic memory for sensory-discriminative components of capsaicin-induced pain: immediate and delayed ratings. Pain. 2009;143(1-2):97-105

40. Kuehn E, Mueller K, Lohmann G, Schuetz-Bosbach S. Interoceptive awareness changes the posterior insula functional connectivity profile. Brain Struct Funct. 2016;221(3):1555-1571.

41. Wiebking C, de Greck M, Duncan NW, Tempelmann C, Bajbouj M, Northoff G. Interoception in insula subregions as a possible state marker for depression: an exploratory fMRI study investigating healthy, depressed and remitted participants. Front Behav Neurosci. 2015;9:82.

42. zu Eulenburg P, Baumgärtner U, Treede RD, Dieterich M. Interoceptive and multimodal functions of the operculo-insular cortex: tactile, nociceptive and vestibular representations. Neuroimage. 2013;83:75-86.

43. Cisler JM, Elton A, Kennedy AP, et al. Altered functional connectivity of the insular cortex across prefrontal networks in cocaine addiction. Psychiatry Res. 2013;213(1):39-46.

44. Terasawa Y, Fukushima H, Umeda S. How does interoceptive awareness interact with the subjective experience of emotion? An fMRI study. Hum Brain Mapp. 2013;34(3):598-612.

45. Mochizuki H, Baumgärtner U, Kamping S, et al. Cortico-subcortical activation patterns for itch and pain imagery. Pain. 2013;154(10): 1989-1998

46. Maihöfner C, Handwerker HO, Birklein F. Functional imaging of allodynia in complex regional pain syndrome. Neurology. 2006;66(5):711-717.

47. Saarela MV, Hlushchuk Y, Williams AC, Schürmann M, Kalso E, Hari R. The compassionate brain: humans detect intensity of pain from another's face. Cereb Cortex. 2007;17(1):230-237.

48. Lamm C, Decety J, Singer T. Meta-analytic evidence for common and distinct neural networks associated with directly experienced pain and empathy for pain. Neuroimage. 2011;54(3):2492-2502.

49. Vachon-Presseau E, Roy M, Martel MO, et al. Neural processing of sensory and emotional-communicative information associated with the perception of vicarious pain. Neuroimage. 2012;63(1):54-62.

50. Deutschländer A, Stephan T, Hüfner K, et al. Imagined locomotion in the blind: an fMRI study. Neuroimage. 2009;45(1):122-128.

51. Kravitz DJ, Peng CS, Baker CI. Real-world scene representations in high-level visual cortex: it's the spaces more than the places. JNeurosci. 2011;31(20):7322-7333.

52. Mullally SL, Maguire EA. A new role for the parahippocampal cortex in representing space. J Neurosci. 2011;31(20):7441-7449.

53. Tracey I, Mantyh PW. The cerebral signature for pain perception and its modulation. Neuron. 2007;55(3):377-391. 
The Journal of Pain Research is an international, peer reviewed, open access, online journal that welcomes laboratory and clinical findings in the fields of pain research and the prevention and management of pain. Original research, reviews, symposium reports, hypothesis formation and commentaries are all considered for publication
The manuscript management system is completely online and includes a very quick and fair peer-review system, which is all easy to use. Visit http://www.dovepress.com/testimonials.php to read real quotes from published authors. 